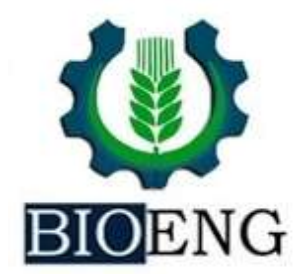

\title{
ATRIBUTOS FÍSICOS DO SOLO E CRESCIMENTO DE RAIZ DA SOJA APÓS CULTIVOS DE OUTONO-INVERNO
}

\author{
L. M. Ribeiro ${ }^{1 *}$,D. S. Flauzino ${ }^{2}$, G. Ceccon $^{3}$
}

${ }^{1}$ UFGD - Universidade Federal da Grande Dourados, Dourados, MS, Brasil
${ }^{2}$ SENAR-MS - Serviço Nacional de Aprendizagem Rural, Dourados, MS, Brasil
${ }^{3}$ Embrapa Agropecuária Oeste - CPAO, Dourados, MS, Brasil

Article history: Received 19 November 2018; Received in revised form 20 February 2019; Accepted 21 February 2019; Available online 30 March 2019.

\section{RESUMO}

Cultivos antecedentes podem modificar as condições físicas do solo, o desenvolvimento e produtividade das culturas. Objetivou-se avaliar os atributos físicos do solo e o crescimento de raízes da soja após cultivos de outono-inverno. O experimento foi conduzido na área experimental da Embrapa Agropecuária Oeste, em Dourados, MS, em LATOSSOLO VERMELHO distroférrico de textura argilosa. O delineamento experimental foi em blocos casualizados com parcelas sub-divididas, com quatro repetições. Nas parcelas foram alocados os quatro cultivos de outono-inverno (Urochloa ruziziensis, consórcio de milho com $U$. ruziziensis, milho safrinha e feijão-caupi (Vigna unguiculata)) e as sub-parcelas as duas cultivares de soja BRS 284 e BRS 360 RR. Foram coletadas amostras de solo e de raízes da soja nas profundidades $0,0-0,10 \mathrm{~m} ; 0,10-0,20 \mathrm{~m} ; 0,20-0,30 \mathrm{~m} ; 0,30-0,40 \mathrm{~m}$. O tipo de cultivo interferiu na qualidade física do solo. O feijão-caupi e $U$. ruziziensis proporcionaram maior microporosidade e porosidade total do solo; o consórcio milho com U. ruziziensis proporcionou maior macroporosidade e porosidade total do solo; o milho safrinha acarretou em maior densidade do solo, mas a soja apresentou maior crescimento de raízes após a $U$. ruziziensis.

Palavras-chave: Integração Lavoura Pecuária; consórcio; compactação do solo.

\section{SOIL PHYSICAL ATTRIBUTES AND SOYBEAN ROOT GROWTH AFTER AUTUMN-WINTER CROPS}

\begin{abstract}
Previous crops may modify soil physical conditions, crop development and yield. The objective was to evaluate soil physical attributes and root growth of soybeans after autumnwinter crops. The experiment was conducted in the experimental area of Embrapa Agropecuária Oeste, in Dourados, MS, in a clayey dystroferric RED LATOSOLO. The experimental design was in randomized blocks with sub-divided plots, with four replications. In the plots were allocated four autumn-winter crops (Urochloa ruziziensis, corn intercropping with $U$. ruziziensis, single corn and cowpea (Vigna unguiculata)) and the subplots the two soybean cultivars BRS 284 and BRS 360 RR. Soil and root samples of soybean were collected at depths of $0.0-0.10 \mathrm{~m} ; 0.10-0.20 \mathrm{~m} ; 0.20-0.30 \mathrm{~m} ; 0.30-0.40 \mathrm{~m}$. The type of crop interfered in the physical quality of the soil. Cowpea and U. ruziziensis provided higher microporosity and total soil porosity; the corn intercropping with $U$.
\end{abstract}

\footnotetext{
*luanmarlon@hotmail.com
} 
ruziziensis provided higher macroporosity and total soil porosity; the single corn increased in soil density, but the soybean showed higher root growth after $U$. ruziziensis.

Keywords: Livestock Integration; intercropping; soil compaction.

\section{INTRODUÇÃO}

O Brasil possui aproximadamente 74 milhões de hectares agricultáveis, e metade dessa área, cultivado em Sistema Plantio Direto (SPD) (FAO, 2017). Porém, em Mato Grosso do Sul, há baixas coberturas do solo pelo uso inadequado do SPD, o que facilita a degradação do solo e, consequentemente, a diminuição da produtividade das culturas (BRUGGEMANN, 2011).

O sucesso desse sistema produtivo deve-se ao fato de que a palhada, acumulada pelas plantas de cobertura de lavouras comerciais ou das pastagens, proporcionam um ambiente favorável à recuperação ou manutenção dos atributos físicos e químicos do solo (MENDONÇA et al., 2013).

A produção de palha pelas culturas em SPD deve aportar de 10 a $20 \mathrm{t} \mathrm{ha}^{-1} \mathrm{de}$ matéria seca por ano de diferentes resíduos vegetais (TRIVELLIN et al., 2013). Além de fornecer uma proteção as camadas

\section{MATERIAIS E MÉTODOS}

O trabalho foi realizado na área experimental da Embrapa Agropecuária Oeste $\left(22^{\circ} 13^{\prime} \mathrm{S}\right.$ e $54^{\circ} 48^{\prime} \mathrm{W}, 480 \mathrm{~m}$ de altitude) em Dourados-MS no ano agrícola 2014/15. O solo foi classificado como LATOSSO VERMELHO Distroférrico, de textura muito argilosa (SANTOS et al., 2013). A análise química do solo foi realizada em 2013, na camada $0-0,20 \mathrm{~m}$, de acordo com metodologia descrita por Silva (2009), apresentando os seguintes resultados: $\mathrm{pH} \mathrm{CaCl} 2=6,2 ; \mathrm{V} \%=79,3 \%$; $\mathrm{MO}=37,9 \mathrm{~g} \mathrm{~kg}^{-1} ; \mathrm{P}=57,3 \mathrm{mg} \mathrm{dm}^{-3} ; \mathrm{K}=$ $1,0 \mathrm{cmol}_{\mathrm{c}} \mathrm{dm}^{-3} ; \mathrm{Ca}=6,5 \mathrm{cmol}_{\mathrm{c}} \mathrm{dm}^{-3} ; \mathrm{Mg}=$ $2,8 \mathrm{cmol}_{\mathrm{c}} \mathrm{dm}^{-3}$ e $\mathrm{Al}=2,7 \mathrm{cmol}_{\mathrm{c}} \mathrm{dm}^{-3}$.

A área experimental é manejada em SPD há 15 anos, num esquema de sucessão de culturas há sete anos, em que no outono-inverno é cultivado feijão-caupi superficiais do solo aos impactos das gotas de chuva (REINERT et al., 2008).

O uso de plantas de cobertura com diferentes sistemas radiculares e relação $\mathrm{C} / \mathrm{N}$ pode favorecer tanto o controle e/ou prevenção da compactação e aumento na porosidade nas camadas superficiais e subsuperficiais do solo (COSTA et al., 2015), estimulando o crescimento radicular das culturas subsequentes (BONFIMSILVA et al., 2012).

Assim, salienta-se que os resíduos vegetais produzidos pelo SPD são indispensáveis para os sistemas produtivos. Pois minimizam os efeitos negativos ao solo promovidos por sistemas agrícolas intensivos (COSTA et al., 2015).

Considerando a necessidade do aprimoramento do SPD visando potencializar os componentes de produção da soja, objetivou-se avaliar os atributos físicos do solo e crescimento de raiz da soja após cultivos de outono-inverno.

(var. guariba - Vigna unguiculata), milho safrinha, Urochloa ruziziensis e consórcio de milho com $U$. ruziziensis, com a soja no verão. Houve pastejo por animais nos tratamentos $U$. ruziziensis a partir de abril e no consórcio milho com $U$. ruziziensis após a colheita do milho, em julho.

O clima da região é classificado como Am (Tropical Monçônico) com verões quentes e invernos secos, temperaturas máximas e chuva excedente nos meses de dezembro e janeiro e temperaturas mínimas e déficit hídrico entre maio e agosto, com precipitação média anual de $1.469 \mathrm{~mm}$, segundo a classificação de Koppen (SANTOS \& SILVA, 2012). Os dados climáticos durante o experimento foram obtidos na Estação Meteorológica da Embrapa 
Agropecuária Oeste, a $300 \mathrm{~m}$ da área experimental (Figura 1).

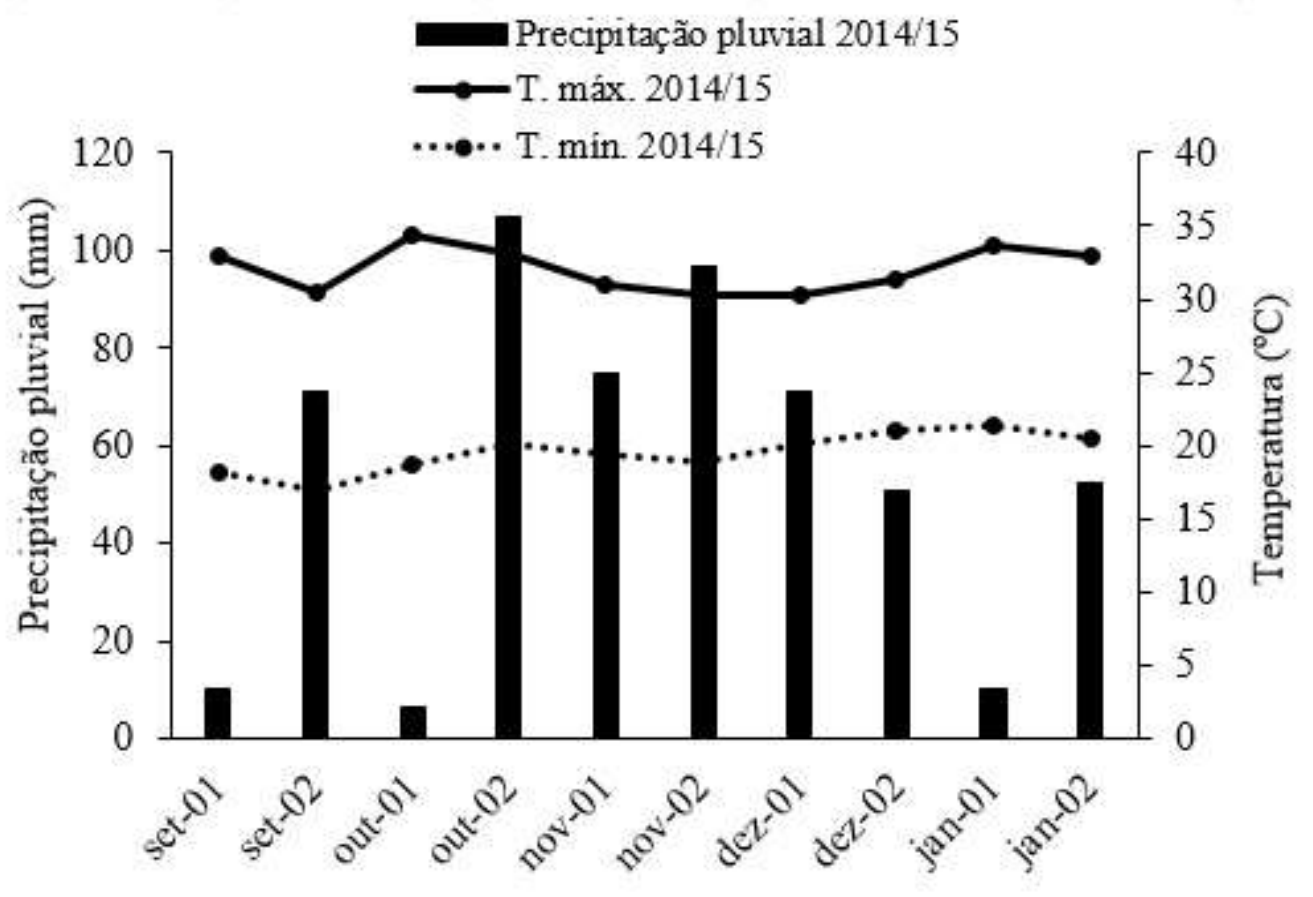

Figura 1. Valores quinzenais de precipitação pluvial, temperaturas máximas e mínimas registradas durante o cultivo da soja-verão 2014/15, em Dourados, MS. Fonte: EMBRAPA (2015).

Para as avaliações dos atributos físicos do solo, as parcelas foram constituídas por quatros cultivos de outono-inverno (feijão-caupi (var. guariba - Vigna unguiculata), milho safrinha, Urochloa ruziziensis e consórcio de milho com $U$. ruziziensis) e as subparcelas pelas camadas do solo $(0,0-0,10 \mathrm{~m}, 0,10-0,20$ $\mathrm{m}, 0,20-0,30 \mathrm{~m}$ e $0,30-0,40 \mathrm{~m})$. Os tratamentos foram arranjados em parcelas subdividas, o delineamento experimental foi em blocos casualizados, com quatro repetições.

Para avaliação do crescimento de raízes de soja foram considerados como fatores de estudo, em que as parcelas foram os cultivos de outono-inverno, as subparcelas as cultivares de soja (BRS 284 e BRS 360 RR), e as camadas de solo, foram consideradas como subsubparcelas.

A soja foi semeada no dia 09/10/2014, em parcelas de sete linhas X seis metros de comprimento em espaçamento de $0,45 \mathrm{~m}$ entre si, utilizandose semeadora modelo PAR 2800 regulada para distribuição de $300 \mathrm{~kg} \mathrm{ha}^{-1}$ da fórmula
NPK 00-20-20, na profundidade de $10 \mathrm{~cm}$, com população de 244.444 plantas ha ${ }^{-1}$, de acordo com recomendações de Tourino et al. (2002). Os tratos culturais foram realizados conforme recomendações técnicas para a cultura.

As coletas de solo foram realizadas entre os dias 23 e 27/11/2014 (estádio R1 da soja). Foram abertas três trincheiras de $1,0 \mathrm{~m} \times 0,40 \mathrm{~m}$ em cada parcela principal, entre a divisa das duas cultivares de soja.

As amostras indeformadas de solo para análise física foram retiradas com anéis volumétricos de $100 \mathrm{~cm}^{3}$ nas camadas de 0,0 - 0,10 m; 0,10 - 0,20 m; $0,20-0,30 \mathrm{~m}$ e $0,30-0,40 \mathrm{~m}$. As amostras foram levadas para análise no laboratório de solos, tecidos vegetais e corretivos, da Embrapa Agropecuária Oeste e determinada a macroporosidade, microporosidade e porosidade total, utilizando o método da mesa de tensão, e a densidade do solo pelo método do anel volumétrico (EMBRAPA, 1997).

Nas trincheiras entre as duas cultivares de soja foram coletadas amostras 
de raízes das cultivares a cada $0,10 \mathrm{~m}$ até $0,40 \mathrm{~m}$ em seguida as raízes foram lavadas em água corrente e armazenadas em solução de álcool 70\%, em câmara fria a $10^{\circ} \mathrm{C}$.

No dia 01/02/2016, avaliou-se a área de superfície radicular, comprimento, diâmetro médio e número de bifurcações das raízes, utilizando-se "Scanner" de leitura ótica (modelo Scanjet4C/T, marca HP), e tiveram suas imagens analisadas

\section{RESULTADOS E DISCUSSÃO}

\section{Atributos físicos do solo}

A análise de variância indicou efeito isolado dos fatores em estudo, constatando-se que os cultivos de outonoinverno influenciaram a macroporosidade, microporosidade, porosidade total e densidade do solo; já a profundidade do solo influenciou a microporosidade, porosidade total e densidade.

O consórcio milho com $U$. ruziziensis apresentou maior Ma e PT com o programa "Win Mac Rhizo" versão 2012b (Regente Instruments Canada Inc.); posteriormente, as raízes foram secas em estufa com circulação de ar forçado a 60 ${ }^{\circ} \mathrm{C}$, para obtenção da massa seca.

Os dados foram submetidos à análise de variância, e quando significativos pelo teste $\mathrm{F}$, as médias foram comparadas pelo teste de Tukey $(\mathrm{p} \leq 0,05)$, utilizando-se $\mathrm{o}$ software SISVAR (FERREIRA, 2011).

(Tabela 1), provavelmente pela menor compactação superficial no consórcio. Isso pode ser decorrente do acúmulo de palha do milho e da braquiária que é maior, possuindo alta densidade de raízes, que após a dessecação (MENDONÇA et al., 2013), aumenta a quantidade de bioporos no solo (CALONEGO \& ROSOLEM, 2010), favorecendo esses atributos físicos.

Tabela 1. Macroporosidade (Ma), microporosidade (Mi), porosidade total (PT) e densidade do solo (DS), durante o cultivo (R1) da soja em Dourados-MS.

\begin{tabular}{ccccc}
\hline \multirow{2}{*}{ Cultivos de outono-inverno } & $\mathrm{Ma}$ & $\mathrm{Mi}$ & $\mathrm{PT}$ & $\mathrm{DS}$ \\
\cline { 2 - 5 } & $\ldots \ldots \ldots \ldots \ldots \ldots .(\%) \ldots \ldots \ldots \ldots \ldots \ldots$ & $\left(\mathrm{kg} \mathrm{dm}^{-3}\right)$ \\
\hline Feijão-caupi & $12,5 \mathrm{ab}$ & $41,5 \mathrm{a}$ & $51,2 \mathrm{~b}$ & $1,24 \mathrm{~b}$ \\
Milho safrinha & $10,1 \mathrm{~b}$ & $41,4 \mathrm{a}$ & $51,6 \mathrm{~b}$ & $1,37 \mathrm{a}$ \\
U. ruziziensis & $10,4 \mathrm{~b}$ & $42,4 \mathrm{a}$ & $52,9 \mathrm{ab}$ & $1,27 \mathrm{~b}$ \\
Média & $14,8 \mathrm{a}$ & $39,2 \mathrm{~b}$ & $54,1 \mathrm{a}$ & $1,19 \mathrm{c}$ \\
Consórcio milho - U. ruziziensis & 12,0 & 41,1 & 52,5 & 1,27 \\
\hline C.V. $(\%)$ & 20,7 & 3,1 & 3,4 & 3,7 \\
\hline
\end{tabular}

Médias seguidas de mesma letra na coluna, não diferem pelo teste de Tukey $(\mathrm{p}<0,05)$.

Os menores valores de Ma no milho safrinha e $U$. ruziziensis solteiros, deve-se possivelmente a redução de macroporos, em função da menor densidade radicular fornecido por essas culturas quando solteiras e ao pastejo dos animais durante o período de pousio, respectivamente.

Quanto a Mi, os cultivos feijãocaupi, milho safrinha e $U$. ruziziensis não diferiram entre si. Esses cultivos antecessores a soja podem incrementar os valores de microporos no perfil do solo, devido ao fato de apresentar menor volume de raiz e palhada sob o solo quando comparado ao consórcio de milho com $U$. ruziziensis (LANZANOVA et al., 2007).

A maior DS sob cultivo de milho safrinha deve-se ao fato de a planta possuir menor quantidade de palha no solo. Já, áreas cultivadas com culturas de outonoinverno, com o passar do tempo tendem a favorecer o aumento da Ma e PT (SÁ et al., 2014). SANTOS et al., (2011) relatam que valores ideais de DS para bom desenvolvimento das culturas em solos argilosos, variam entre 1,10 a $1,60 \mathrm{~kg} \mathrm{dm}^{-3}$, 
isto é, demonstrando que mesmo com cultivo de milho safrinha, os valores foram adequados para o desenvolvimento das culturas subsequentes.

Os maiores valores de $\mathrm{Mi}$ observados nas profundidades de 0,0 - 0,10 $\mathrm{m}$ e 0,30 - $0,40 \mathrm{~m}$ (Tabela 2) possivelmente, é consequência da menor resistência à penetração do solo, pois nessas profundidades os valores de porosidade total também são maiores. As camadas intermediárias podem apresentar aumento na densidade do solo, visto que o acúmulo dos resíduos vegetais em SPD e a umidade do solo nas camadas superficiais é maior, propiciando condições que favoreçam o crescimento radicular, e consequentemente maior $\mathrm{Mi}$ e PT (BARRIOS et al., 2006).

Tabela 2. Microporosidade (Mi), porosidade total (PT) e densidade de solo (DS), nas profundidades do solo, em Dourados-MS.

\begin{tabular}{cccc}
\hline \multirow{2}{*}{ Profundidades } & $\mathrm{Mi}$ & $\mathrm{PT}$ & $\mathrm{DS}$ \\
\cline { 2 - 4 } & $\ldots \ldots \ldots \ldots(\%) \ldots \ldots \ldots \ldots \ldots \ldots \ldots \ldots$ & $\left(\mathrm{kg} \mathrm{dm}^{-3}\right)$ \\
\hline $0,00-0,10 \mathrm{~m}$ & $42,1 \mathrm{a}$ & $53,8 \mathrm{ab}$ & $1,27 \mathrm{a}$ \\
$0,10-0,20 \mathrm{~m}$ & $40,6 \mathrm{bc}$ & $52,0 \mathrm{~b}$ & $1,29 \mathrm{a}$ \\
$0,20-0,30 \mathrm{~m}$ & $40,3 \mathrm{c}$ & $52,4 \mathrm{~b}$ & $1,27 \mathrm{a}$ \\
$0,30-0,40 \mathrm{~m}$ & $41,8 \mathrm{ab}$ & $54,6 \mathrm{a}$ & $1,19 \mathrm{~b}$ \\
Média & 41,2 & 53,2 & 1,26 \\
\hline C.V.\% & 3,0 & 3,4 & 3,7 \\
\hline
\end{tabular}

Médias seguidas de mesma letra na coluna, não diferem pelo teste de Tukey $(\mathrm{p}<0,05)$.

Os maiores valores de DS foram observados nas profundidades $0,0-0,10$ m; $0,10-0,20 \mathrm{~m}$ e $0,20-0,30 \mathrm{~m}$ (Tabela 2). Esses resultados podem estar relacionados com pisoteio de animais nos tratamentos de $U$. ruziziensis e no consórcio de milho com $U$. ruziziensis, dessa forma, podendo influenciar maior DS nas camadas superficiais e subsuperficiais do solo. Fidalski et al. (2013) avaliando atributos físicos do solo após pastejo contínuo, observaram maiores valores de DS nas profundidades 0,0 - 0,10 $\mathrm{m} ; 0,10-0,20 \mathrm{~m}$ e $0,20-0,30 \mathrm{~m}$, atribuindo esses resultados à presença sistemática dos animais, semelhantes aos deste trabalho.

\section{Crescimento de raiz}

As características das raízes de soja foram influenciadas pelos fatores em estudo de forma isolada. Ambas cultivares após cultivo de $U$. ruziziensis apresentaram maior CR, AR, NB e MSR em comparação aos demais sistemas (Tabela 3). Isso pode estar relacionado com a física do solo, pois nesse mesmo cultivo, houve maior Mi, PT e menor DS (Tabela 1), o que favoreceu o crescimento do sistema radicular da cultura.

Tabela 3. Comprimento de raiz (CR), área superficial de raiz (AR), número de bifurcações (NB) e massa seca de raiz (MSR), de soja após cultivos de outono-inverno, em Dourados-MS.

\begin{tabular}{ccccc}
\hline \multirow{2}{*}{ Cultivos de outono inverno } & $\mathrm{CR}$ & $\mathrm{AR}$ & $\mathrm{NB}$ & $\mathrm{MSR}$ \\
\cline { 2 - 5 } & $(\mathrm{cm})$ & $\left(\mathrm{cm}^{2}\right)$ & - & $\left(\mathrm{kg} \mathrm{cm}^{3}\right)$ \\
\hline Feijão-caupi & $98,8 \mathrm{~b}$ & $15,0 \mathrm{~b}$ & $368 \mathrm{ab}$ & $0,07 \mathrm{~b}$ \\
Milho safrinha & $88,2 \mathrm{~b}$ & $14,6 \mathrm{~b}$ & $296 \mathrm{~b}$ & $0,16 \mathrm{ab}$ \\
U. ruziziensis & $149,0 \mathrm{a}$ & $25,4 \mathrm{a}$ & $645 \mathrm{a}$ & $0,31 \mathrm{a}$ \\
Consórcio milho - U. ruziziensis & $96,8 \mathrm{~b}$ & $16,8 \mathrm{~b}$ & $368 \mathrm{ab}$ & $0,17 \mathrm{ab}$ \\
Média & 108,2 & 18,0 & 419,3 & 0,17 \\
\hline C.V. $(\%)$ & 60,6 & 62,0 & 87,4 & 56,2 \\
\hline
\end{tabular}

Médias seguidas de mesma letra na coluna, não diferem pelo teste de Tukey $(\mathrm{p}<0,05)$. 
A soja em sucessão ao milho safrinha e feijão-caupi, apresentou menor $\mathrm{CR}, \mathrm{AR}$,

NB e MSR, provavelmente pela menor Ma e maior Mi nestes cultivos. Genro Junior et al. (2009) relatam que a menor Ma, é responsável, por impedimentos físicos que são limitantes para o crescimento das raízes, podendo também diminuir a quantidade de água armazenada no solo. No entanto, as respostas em crescimento da raiz podem variar, Pivetta et al. (2011) avaliando o sistema de produção sobre o crescimento e atividade de raiz de soja, observaram maior comprimento de raiz em sucessão à gramíneas.

No que refere-se às profundidades, a soja apresentou maior CR, AR, NB e MSR na camada de 0,0 - 0,10 m, diferindo-se apenas da camada 0,20 - 0,30 m (Tabela 4). Nessa camada houve maior Mi, fazendo com que as raízes alterassem seu mecanismo de exploração, isto é, pode ter ocorrido modificações, tais como a emissão de novas raízes com menor diâmetro (raízes finas), que consequentemente aumentaram o NB (CONCEIÇÃO et al., 2005), visando maior exploração na rizosfera.

Isso ocorre porque em camadas mais adensadas e menos porosas pode reduzir ou até impedir o crescimento radicular (SOUZA et al., 2006). Após essas conseguirem ultrapassar a camada com maior DS, as mesmas voltaram a apresentar maior diâmetro nas camadas intermediárias $(0,20-0,30 \mathrm{~m})$ (Tabela 4).

Tabela 4. Comprimento de raiz (CR), área superficial de raiz (AR), diâmetro médio de raiz (DR), número de bifurcações (NB) e massa seca de raiz (MSR) de soja nas profundidades do solo após cultivos de outono-inverno, em Dourados-MS.

\begin{tabular}{cccccc}
\hline \multirow{2}{*}{ Profundidades } & CR & AR & DR & NB & MSR \\
\cline { 2 - 6 } & $(\mathrm{cm})$ & $\left(\mathrm{cm}^{2}\right)$ & $(\mathrm{mm})$ & - & $\left(\mathrm{kg} \mathrm{cm}^{3}\right)$ \\
\hline $0,00-0,10 \mathrm{~m}$ & $277,9 \mathrm{a}$ & $41,7 \mathrm{a}$ & $0,4 \mathrm{~b}$ & $1.200 \mathrm{a}$ & $0,59 \mathrm{a}$ \\
$0,10-0,20 \mathrm{~m}$ & $58,3 \mathrm{~b}$ & $10,5 \mathrm{~b}$ & $0,7 \mathrm{ab}$ & $221 \mathrm{~b}$ & $0,06 \mathrm{~b}$ \\
$0,20-0,30 \mathrm{~m}$ & $54,7 \mathrm{~b}$ & $11,5 \mathrm{~b}$ & $0,8 \mathrm{a}$ & $145 \mathrm{~b}$ & $0,03 \mathrm{~b}$ \\
$0,30-0,40 \mathrm{~m}$ & $41,7 \mathrm{~b}$ & $8,1 \mathrm{~b}$ & $0,6 \mathrm{ab}$ & $111 \mathrm{~b}$ & $0,02 \mathrm{~b}$ \\
Média & 108,2 & 18,0 & 0,6 & 419,3 & 0,18 \\
\hline C.V. $(\%)$ & 60,5 & 62,0 & 75,2 & 87,4 & 56,1 \\
\hline
\end{tabular}

Médias seguidas de mesma letra na coluna, não diferem pelo teste de Tukey $(\mathrm{p}<0,05)$.

\section{CONCLUSÃO}

Os cultivos de outono-inverno promoveram mudanças nos atributos físicos do solo.

A Urochloa ruziziensis proporcionou maior crescimento de raízes de soja.

\section{REFERÊNCIAS BIBLIOGRÁFICAS}

BARRIOS, M. B.; BOZZO, A. A.; DEBELIS, S. P.; PEREYRA, A. M.; BUJÁN, A. Soil physical properties and root activity in a soybean second crop/maize rotation under direct sowing and conventional tillage. Spanish Journal of Agricultural Research, v.4 (4): 355362, 2006.
O milho safrinha acarretou maiores valores de microporosidade e densidade do solo.

BONFIM-SILVA, E. M.; VALADÃO JUNIOR, D. D.; REIS, R. H. P.; CAMPOS, J. J.; SCARAMUZZA, W. L. M. P. Establishment of Xaraés and Marandu grasses under levels of soil compaction. Revista Brasileira de 
Engenharia Agrícola e Ambiental, v.32 (4): 727-735, 2012.

BRUGGEMANN, G. Estado da arte e divulgação do Plantio Direto no Brasil. Revista Plantio Direto, v.20 (122): 16-23, 2011.

CAlONEGO, J. C.; ROSOlEM, C. A. Soybean root growth and yield in rotation with cover crops under chiseling and notill. European Journal of Agronomy, v.33 (11): 242-249, 2010.

CONCEIÇÃO, P. C.; AMADO, T. J. C.; MIELNICZUK, J.; SPAGNOLLO, E. Qualidade do solo em sistemas de manejo avaliada pela dinâmica da matéria orgânica e atributos relacionados. Revista Brasileira de Ciência do Solo, v.29 (5): 777-788, 2005.

COSTA, N. R.; ANDREOTTI, M.; LOPES, K. S. M.; YOKOBATAKE, K. L.; FERREIRA, J. P.; PARIZ, C. M.; BONINI, C. S. B.; LONGHINI, V. Z. Atributos do Solo e Acúmulo de Carbono na Integração Lavoura-Pecuária em Sistema Plantio Direto. Revista Brasileira de Ciências do Solo, v.39 (3): 852-863, 2015.

EMBRAPA - Empresa Brasileira de Pesquisa Agropecuária. Guia clima. Brasília. Dourados-MS: Embrapa Agropecuária Oeste, 2017. Disponível em: $<$ http://www.cpao.embrapa.br/clima/?lc=si te/banco-dados/construtor-basico $>$. Acesso em: 18 nov. 2018.

EMPRESA BRASILEIRA DE PESQUISA AGROPECUÁRIA - EMBRAPA. Centro Nacional de Pesquisa de Solos. Manual de métodos de análise de solo. Rio de Janeiro, 1997. 212p.

FAO - Organização das Nações Unidas para Agricultura e Alimentação. Perspectivas Agrícolas no Brasil: desafios da agricultura brasileira 20152014. França-FR, 2017. Disponível em:
<http://www.fao.org.br/download/PA2014 2015CB.pdf>. Acesso em: 16 nov. 2018.

FERREIRA, D. F. Sisvar: a computer statistical analysis system. Ciência \& Agrotecnologia, v.35 (6): 1039-1042, 2011.

FIDALSKI, J.; TORMENA, C. A.; ALVES, S. J. Intervalo hídrico ótimo de um Latossolo distrófico, após o primeiro período de pastejo contínuo de Brachiaria ruziziensis, em sistema Integração Lavoura-Pecuária. Revista Brasileira de Ciência do Solo. v.37 (3): 775-783, 2013.

GENRO JUNIOR, S. A.; REINERT, D. J.; REICHERT, J. M.; ALBUQUERQUE, J. A. Atributos físicos de um Latossolo vermelho e produtividade de culturas cultivadas em sucessão e rotação. Ciência Rural, v.39 (1): 65-73, 2009.

LANZANOVA, M. E.; NICOLOSO, R. D. S.; LOVATO, T.; ELTZ, F. L. F.; AMADO, T. J. C.; REINERT, D. J. Atributos físicos do solo em sistema de integração lavoura-pecuária sob plantio direto. Revista Brasileira de Ciência do Solo, v.31 (1): 1131-1140, 2007.

MENDONÇA, V. Z.; MELLO, L. M. M.; ANDREOTTI, M.; PEREIRA, F. C. B. L.; LIMA, R. C.; VALÉRIO FILHO, W. V.; YANO, E. H. Avaliação dos atributos físicos do solo em consórcio de forrageiras, milho em sucessão com soja em região de Cerrados. Revista Brasileira de Ciência do Solo, v.37 (1): 251-259, 2013.

PIVETTA, L. A.; CASTOLDI, G.; SANTOS, G. P.; ROSOLEM, C. A. Crescimento e atividade de raíz de soja em função do sistema de produção. Pesquisa Agropecuária Brasileira, v.46 (11): 15471554, 2011.

REINERT, D. J.; ALBUQUERQUE, J. A.; REICHERT, J. M.; AITA, C.; ANDRADA, M. M. C. Limites críticos de densidade do solo para o crescimento de raízes de plantas de cobertura em 
Argissolo Vermelho. Revista Brasileira de Ciência do Solo, v.32 (5): 1805-1816, 2008.

SÁ, J. C. M.; TIVET, F.; LAL, R.; BRIEDIS, C.; HARTMAN, D. C.; SANTOS, J. C.; SANTOS, J. B. Longtermtillage systems impacts on soil $\mathrm{C}$ dynamics, soil resilience and agronomic productivity of a Brazilian Oxisol. Soil and Tillage Research, v.136 (1): 38-50, 2014.

SANTOS, V. A.; SILVA, C. A. O clima de Dourados (MS) e a proposição de um roteirometodológico simples para entender a poluição atmosférica de áreas urbanas. Revista Geonorte, v.3 (5): 969-982, 2012.

SANTOS, G. G.; SILVA, E. M.; MARCHÃO, R. L.; SILVEIRA, P. M.; BRUAND, A.; JAMES, F.; BECQUER, T. Analysis of physical quality of soil using the water retention curve: Validity of the S-index. Comptes Rendus Geoscience, v.343 (4): 295-301, 2011.

SANTOS, H. G.; JACOMINE, P. K. T.; ANJOS, L. H. C.; OLIVEIRA, V. A.; LUMBRERAS, J. F.; COELHO, M. R.; ALMEIDA, J. A.; CUNHA, T. J. F.; OLIVEIRA, J. B. Sistema brasileiro de classificação de solos. Rio de Janeiro: Embrapa Solos, 2013. 353p.

SILVA, F. C. Manual de análises químicas do solo, plantas e fertilizantes. Brasília, DF: Embrapa Informação Tecnológica, 2009. 627p.

SOUZA, E. D.; CARNEIRO, M. A. C.; PAULINO, H. B.; SILVA, C. A.; BUZETTI, S. Frações do carbono orgânico, biomassa e atividade microbiana em um Latossolo Vermelho sob Cerrado submetido a diferentes sistemas de manejos e usos do solo. Acta Scientiarum Agronomy, v.28 (3): 323-329, 2006.

TOURINO, M. C. C.; REZENDE, P. M.; SALVADOR, N. Espaçamento, densidade e uniformidade de semeadura na produtividade e características agronômicas da soja. Pesquisa Agropecuária Brasileira, v.37 (8): 10711077, 2002.

TRIVELLIN, P. C. O.; FRANCO, H. C. J.; OTTO, R.; FERREIRA, D. A.; VITTI, A. C.; FORTES, C.; FARONI, C. E.; OLIVEIRA, E C. A.; CANTARELLA, H. Impact of sugarcane trash on fertilizer requirements for São Paulo, Brazil. Scientia Agricola, v.70 (5): 345-352, 2013. 